\title{
OBSERVATIONS ON THE WATER CONTENT OF BONE
}

\author{
J. W. Smith, St Andrews, Scotland
}

From the Department of Anatomy, St Salvator's College, St Andrews

In relation to its water content whole mammalian bone may be considered conveniently as two divisions. The lacunae, the canaliculi and the vascular spaces are unossified, and have been described collectively by Robinson (1960) as the marrow-vascular-osteocyte or M.V.O. space. The lacunae and canaliculi account, fairly constantly, for about one-tenth of the total volume of any region of bone (Robinson 1960), but the vascular spaces vary widely in size from the large cavities characteristic of developing and of osteoporotic bone to the narrow tubular spaces of mature bone. About nine-tenths of the total volume of the marrow-vascularosteocyte space consists of water, and it is probable that this figure is independent of both the character and age of the bone.

The second division of whole bone is the calcified tissue which is located around and between the elements of the marrow-vascular-osteocyte space, and which will be referred to here as bone tissue proper. In the simplest chemical terms it consists of inorganic, organic and water fractions, the latter being divisible into three parts, namely the water of inorganic crystallisation, collagen-bound water, and free water.

Bone tissue proper is seldom homogeneous: indeed, because of the nature of both primary osteogenesis and secondary resorption and replacement, most fields are aggregates of dissimilar microscopic regions in which the three chemical fractions are combined in different proportions.

The demonstration of regional differences in the concentration of inorganic material in bone tissue proper by microradiography is now commonplace, and much is already understood of the relationship of such differences to the process of osteogenesis and the age of the tissue. Regional variations in the amount of organic material in bone tissue proper may be studied at the microscopic level by various methods including that of carbonisation (Smith 1963). The water fraction of bone tissue proper has always been the most difficult to assess. Even the determination of the water content in a macroscopic region is beset with difficulties: the proportion of bone tissue proper in the block of whole bone must be determined, and the evaporation of water from the tissue and the absorption of water from the atmosphere have to be controlled throughout the preparation of the block and subsequent procedures. Even then the method gives no information of any differences which may exist in the water contents of the dissimilar microscopic regions of which the macroscopic block is composed. This paper describes a method which, though it fails to measure the absolute amount of water in the tissue, does give a useful indication of the relative water contents in both the dissimilar microscopic subdivisions of bone tissue proper and the various elements of the marrowvascular-osteocyte space. The information obtained by this method has been correlated with that derived from microradiographs and carbonised preparations.

\section{METHODS}

Water content-Either fresh or fixed bone is ground between glass plates to a thickness of approximately 100 microns, and thereafter the section is placed between two glass slides, and dried to constant weight at 105 degrees Centigrade. It has been demonstrated (Smith and Walmsley 1959) that in bone the process of drying is associated with a minute contraction of the tissue and, since this change is reversed on rehydration, it is probable that the internal stresses which cause the contraction are due to the heating of the collagen fraction above its shrinkage temperature of 64 degrees Centigrade. However, the contraction in any one 
dimension is only of the order 0.15 per cent (Smith and Walmsley 1959); so in the present context it may be ignored.

Because of this almost complete rigidity the water which is lost from the section is replaced by an air space. It is this air space which can be demonstrated by the method under discussion, and it is therefore essential that its volumetric relationship to the original water space in the section should be defined as closely as possible. It is considered that drying to constant weight at 105 degrees Centigrade does not remove the water of crystallisation of the mineral fraction of the tissue. But since the amount of this water is small (Neuman, Toribara and Mulryan 1953), and since it is not directly associated with ionic movement through the tissue, failure to demonstrate it is no great disadvantage. On the other hand the treatment does remove the other two parts of the total water fraction. The free water of the tissue, including the crystal hydration shell (Neuman and Neuman 1958) is completely removed, and there seems no reason to doubt that it is replaced by an air space which is equal to it, volume for volume. The collagen-bound water is also completely removed, but the air space which replaces it is not of equal volume. Because the partial specific volume of collagen is about 0.75 cubic centimetre per gramme (Edsall 1953) the air space is appreciably less than that of the water it replaces. Thus the air space which this method demonstrates is not equal to the total volume of the water compartment, nor is it directly proportional to it; rather it is a function of the water compartment which is dependent in some measure on the concentrations of both inorganic and organic material within the tissue.

The subsequent stages of the method consist essentially in refilling the air space with a solution of an inert pigment, precipitating the pigment in situ, and clearing the section so that it can be examined under the microscope. The density of the pigment is then proportional to the size of the air space and is consequently a function of the original water content. Variations in water content in adjacent microscopic regions are thus indicated by differences in colour density.

Two solutions have been employed successfully. Some of the sections were immersed, immediately after drying, and while still hot, in a saturated solution of Sudan 4 in xylol. After forty-eight hours they were removed and the pigment was precipitated in the section by evaporation of the solvent overnight at 37 degrees Centigrade. The section was then gently reground to remove surface deposits, and cleared and mounted in glycerine jelly (Fig. 7). Because, in relation to cortical bone, Sudan 4 is inert, the pigment found in the cleared sections may be regarded confidently as occupying the original water space. Other sections were immersed in a saturated solution of iodine in absolute alcohol. After forty-eight hours they were removed and the iodine was precipitated by allowing the alcohol to evaporate at room temperature. The sections were then reground and cleared and mounted in glycerine jelly (Fig. 5). Iodine impregnation has certain disadvantages compared with impregnation by Sudan 4. lodine is not completely inert in relation to bone for it combines with tyrosine and phenylallanine which constitute together about 3.5 per cent of the amino acid complement of collagen (Tristram 1953, Eastoe 1955). However, the products of these reactions are both colourless, and since the method depends on colour differences in the cleared section they are not significant. Secondly, iodine is slightly soluble in water and also sublimates readily at room temperature. As a result the sections prepared by this method tend to fade rather rapidly. Inorganic content-Once the water content of a ground bone section has been demonstrated by the above method, and recorded either by colour or black and white photography, the iodine or the Sudan 4 was removed from the section by immersion in an excess of the relevant solvent. The dried section was then submitted to microradiography to demonstrate variations in the inorganic content of the tissue. In the present investigation this was carried out with a Phillips Contact Microradiographic Unit No. 5 and Kodak Experimental Film (Emulsion V.6078). No attempt was made to make an absolute assessment of inorganic concentrations, because, as already noted, the assessment of water content is itself only relative. 
Organic content-After microradiography the organic content of the same section was demonstrated by carbonisation (Smith 1963). The ground sections were placed between two glass slides and heated on an asbestos wire grill until they turned golden yellow in colour. The heat was then removed, and by the time the slides were cool the sections appeared dark brown to black. After immersion in xylol the sections were cleared and mounted in balsam. This process reduces the organic constituents of the tissue to carbon, while the original form of the section is maintained by the rigidity of the inorganic fraction. The original organic content of any area in the section is proportional to the carbon density, so that areas with a high organic content appear dark while those with a low organic content appear light.

\section{OBSERVATIONS}

Secondary bone-Figure 1 is a fairly high power microradiograph of a ground section of human secondary bone which shows parts of two secondary osteones. In the osteone in the upper part of the field (A) the mineral content, as indicated by the $\mathrm{x}$-ray absorption, is much

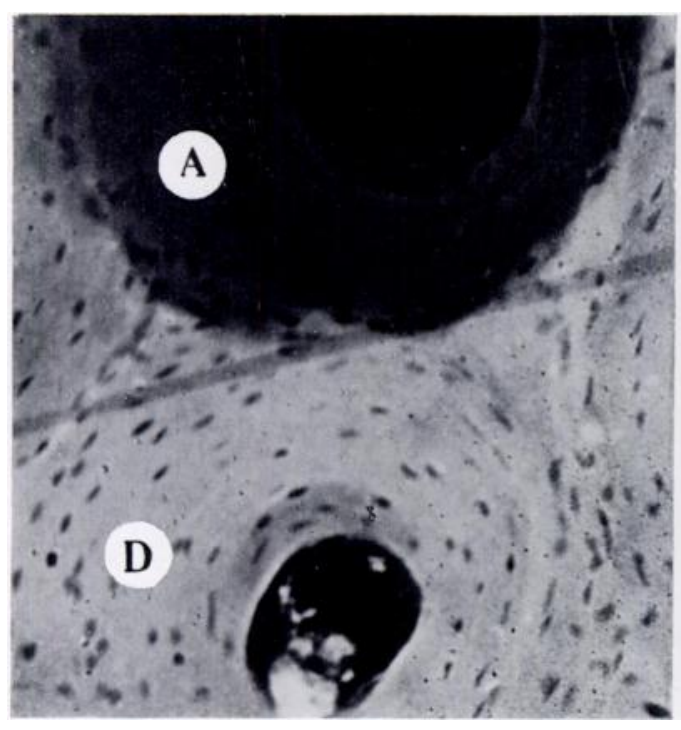

FIG. 1

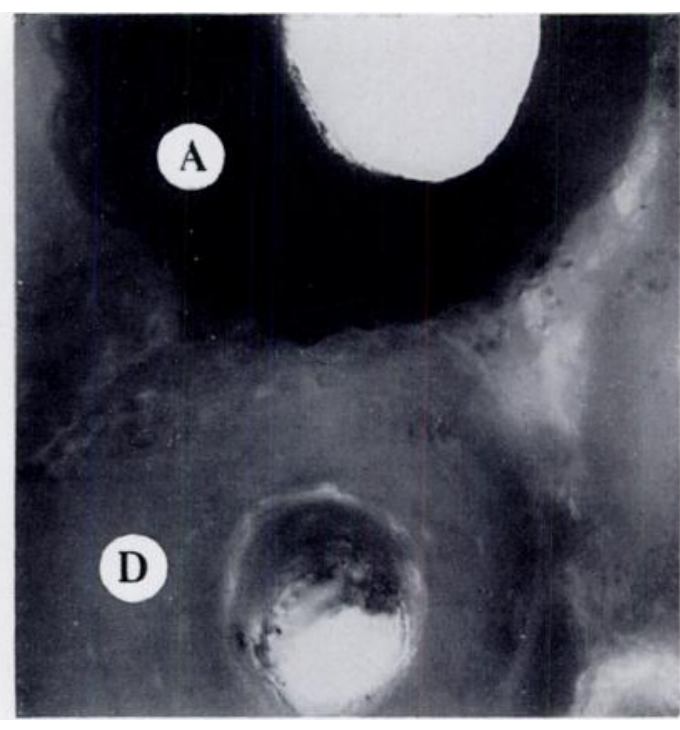

Fig. 2

Figure 1-Microradiograph of a ground section of adult human tibia. $A=$ young secondary osteone. $D=$ older secondary osteone. ( 220.) Figure 2-A carbonised preparation of the same region as in Figure $1 . A=$ young secondary osteone. $\mathrm{D}=$ older secondary osteone. $(\times 220$.)

lower than that in the rest of the field: moreover, it is practically uniform from the outer to the inner border and there is no mineral gradient and no marked edge sclerosis. The vascular canal is very wide and has a diameter of about 140 microns compared to an average definitive diameter in human bone of about 30 microns. All these features indicate that this is a young osteone which is still in the process of formation and in which the process of mineralisation is at an early stage. On the other hand, in the osteone in the lower part of the field (D) the mineral content is considerably higher and the diameter of the vascular canal is approaching definitive dimensions. And, although these features give no indication of the absolute age of this region, they do indicate that osteone $\mathrm{D}$ is considerably older than osteone $\mathrm{A}$.

Figure 2 is a carbonised preparation of the same field as that illustrated in Figure 1. It shows that the degree of blackening, or the carbon density, is much greater in osteone A than in osteone $\mathrm{D}$ and consequently that the organic content was higher in the younger than in the older osteone. In Figure 5 the water content of the same region is indicated by iodine impregnation. In the older osteone $\mathrm{D}$ the lacunae, the canaliculi and the vascular canal, 
which together make up the marrow-vascular-osteocyte space, are heavily impregnated. This degree of impregnation indicates the high water content of these regions, and although the canaliculi are not as clearly demonstrated as they are in a decalcified section stained by Mulligan's $(1958,1964)$ method* (Fig. 3), this is due, in large measure, to the comparatively low translucency of an undecalcified, 100 micron section such as that in Figure 5. In the same osteone (D) the bone tissue proper, which lies around and between the elements of the marrowvascular-osteocyte space, contains little pigment, and it is considered that this shows that the water content of this tissue is correspondingly low. In the younger osteone (A) the lacunae and the wide vascular space are again deeply pigmented, but the canaliculi are largely obscured by the dark coloration of the surrounding bone tissue proper. Thus although the water content of the marrow-vascular-osteocyte space is similar in the two osteones, the water content of the bone tissue proper is much higher in osteone $\mathrm{A}$ than in osteone $\mathrm{D}$.

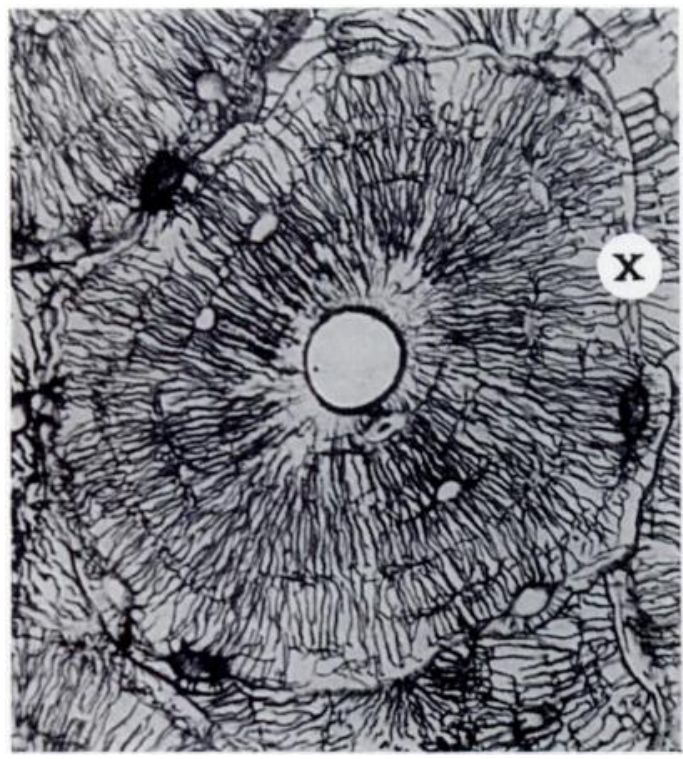

Fig. 3

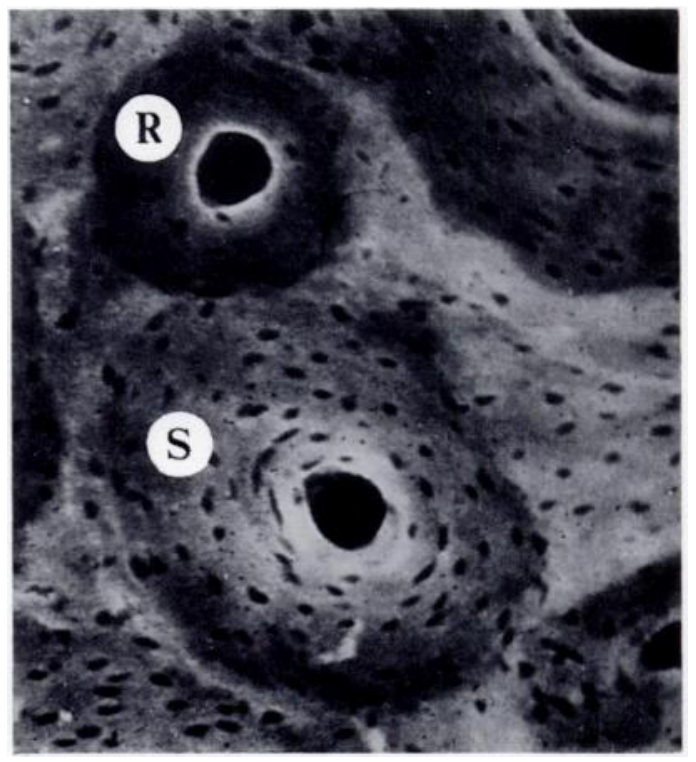

FIG. 4

Figure 3-A transverse section of decalcified adult human femur stained by Mulligan's silver method. $X=$ the reversal line. $(\times 190$.) Figure $4-$ Microradiograph of a ground section of an adult human femur. $R=$ osteone exhibiting edge sclerosis. $\mathbf{S}=$ osteone exhibiting mineral gradient. $(\times 200$.

Thus in the young and incomplete osteone $\mathrm{A}$ a low inorganic concentration in the bone tissue proper is associated with a high organic content and a high water content, whereas, conversely, in the older osteone $\mathrm{D}$ a higher inorganic concentration in the bone tissue proper is associated with lower organic and water contents.

A similar iodine preparation is illustrated in Figure 6 at a lower magnification. In the relatively young osteone (M) the water content, as demonstrated by the iodine coloration, is high near the periphery and somewhat less towards the centre. It is well known that during the mineralisation of secondary osteones a high central concentration of mineral, either in the form of edge sclerosis (Fig. $4 \mathrm{R}$ ) or as a centripetal mineral gradient (Fig. $4 \mathrm{~S}$ ), is a frequent occurrence. It is probable, therefore, that the distribution of water in osteone $M$ in Figure 6 is to be correlated with a mineral distribution of this kind.

* Mulligan's method-Place frozen sections of formalin fixed, decalcified bone in fifty millilitres of 1 per cent silver nitrate and fifty drops of Pyridine at 45 degrees Centigrade for three hours. Transfer to equal parts of the same solution and a reducing solution consisting of equal parts 1.5 per cent Analar Formalin and 10 per cent sodium sulphite. The reduction must be controlled microscopically. Transfer to 5 per cent sodium thiosulphite for one minute. Wash in distilled water for one hour. Mount on gelatinised slides (Mulligan 1964). 
Around osteone $\mathbf{M}$ in Figure 6 are parts of other osteones. All these are older than $\mathbf{M}$ because each has been peripherally eroded by the resorption cavity in which $\mathrm{M}$ was formed. In conformity with this greater age their iodine content, and therefore their water content, is less. The osteone in the lower right quadrant of the field is surrounded by a reversal line (NN),

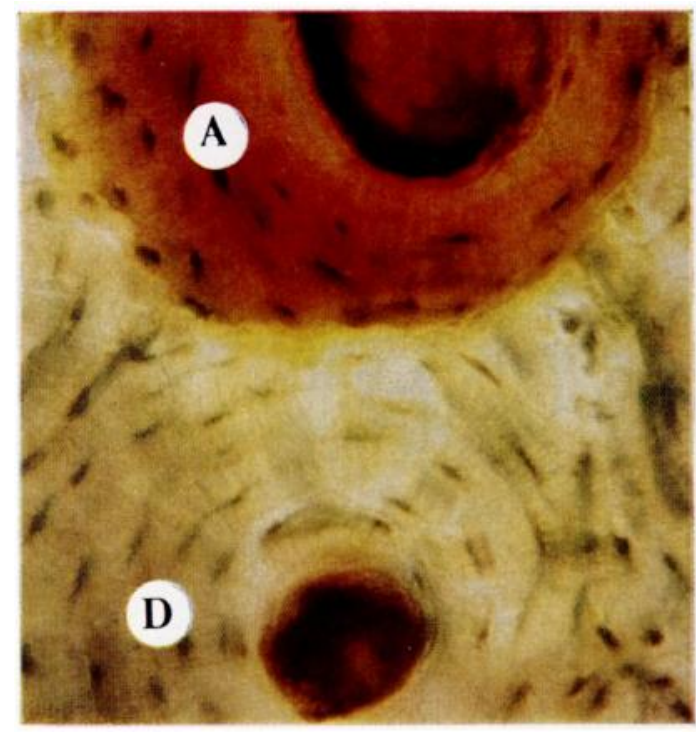

FIG. 5

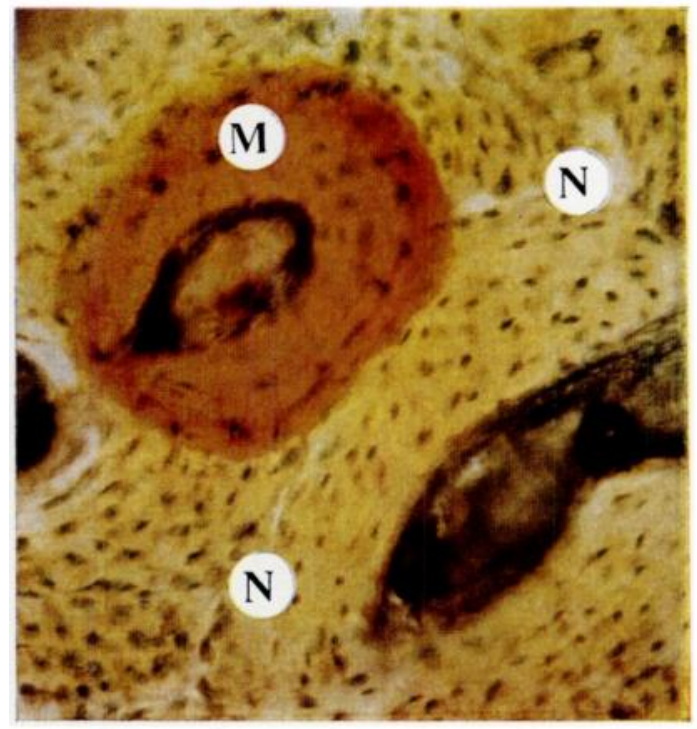

FIG. 6

Figure 5-Iodine impregnation of the region shown in Figures 1 and 2. $A=$ young secondary osteone. $D=$ older secondary osteone. ( $\times 220$.) Figure 6-Iodine impregnation of a ground section of an adult human femur. $\mathbf{M}=$ young secondary osteone. $\mathbf{N}=$ reversal line. $(\times 150$.

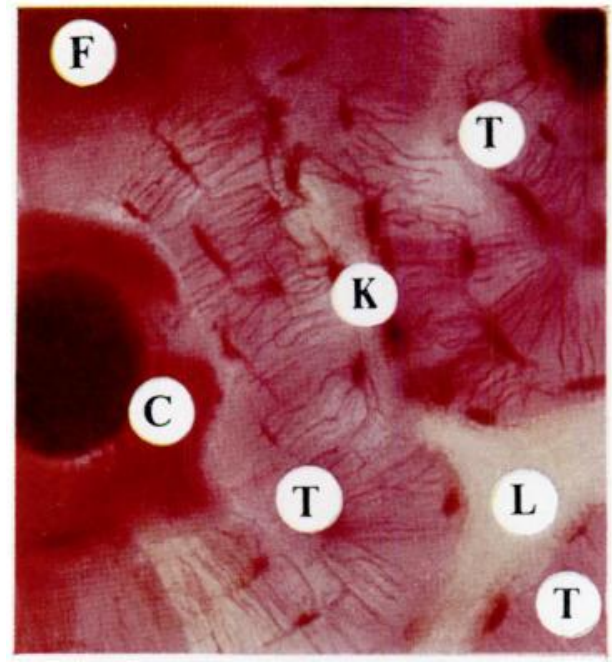

Fig. 7

Sudan 4 impregnation of a ground section of an adult human tibia. $\mathrm{C}$ and $\mathrm{F}$-young secondary osteones. T T T =older secondary osteones. $\mathrm{K}=$ reversal line. $\mathrm{L}=$ interstitial area. $(\times 250$.)

the middle part of which has been destroyed by the resorption cavity associated with osteone $\mathrm{M}$. It is well known that such a reversal line has a very high mineral content, and it has also been demonstrated that in carbonised preparations the organic concentration is very low (Smith 
1963). Furthermore, as shown in Figure 3, a reversal line is traversed by few of the canaliculi which pervade the main part of the osteone. The complete lack of iodine in the reversal line in Figure 6 confirms this paucity of canaliculi and indicates a very low water content in this region of bone tissue proper.

Figure 7 is a high power view of an area of secondary bone which has been impregnated with Sudan 4. In the canaliculi and lacunae in the regions TTT, and in the vascular canal in the upper right corner, the high water content is indicated by dense impregnation. The bone tissue proper in the same regions is coloured light pink and therefore contains a moderate amount of water. The osteone $\mathrm{C}$ has a wide vascular canal and it is evident that it is very young in comparison to the other regions. Here the canaliculi and lacunae are largely obscured by a dense aggregation of pigment, much as they were in osteone $\mathrm{A}$ in Figure 5, and this dense pigmentation indicates the high water content of the bone tissue proper in this young region. A similar region of high water content is apparent at $\mathrm{F}$. The region $\mathrm{L}$ contains very little if any pigment. The peripheral part of this region consists of the reversal lines surrounding the osteones TTT which join together in the region $\mathrm{K}$. On the other hand the central part of region $\mathrm{L}$ is the oldest in the field and consists of an old fully calcified interstitial area. Thus

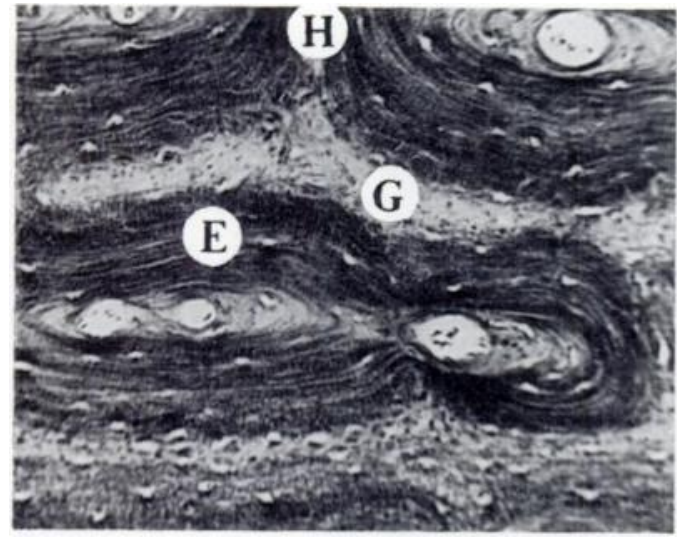

FIG. 8

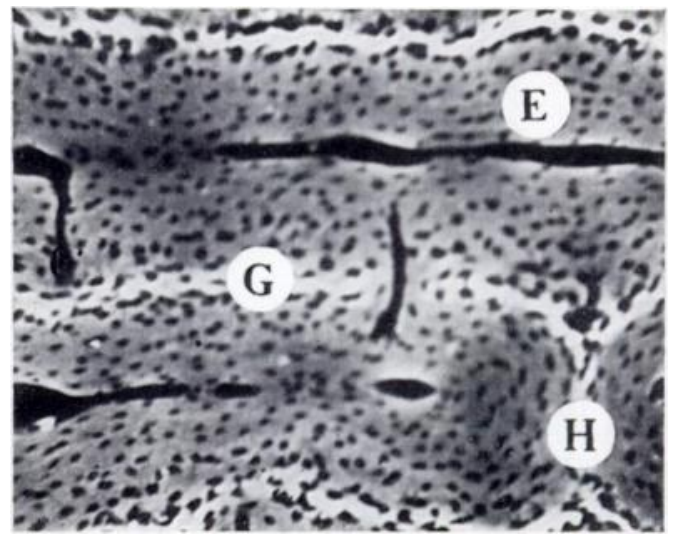

FIG. 9

Figure 8-Transverse section of a decalcified radius of a horse. $E=$ primary osteone. $G$ woven-fibred plaque. $H$ radial plaque. (Weidenreich, 110.) Figure 9-Microradiograph of a ground section of the femur of a pig, labelled as in Figure 8. (110.)

a low water content and absences of canaliculi are not restricted to reversal lines: the same features are associated with very old and fully calcified regions of interstitial bone. Indeed, the absence of canaliculi in such regions has been noted by other techniques by Mulligan (1958), Frost (1960a) and Sissons (1962).

Primary bone--Primary osteone bone constitutes a large part of the skeleton of many animals, and is a feature of the early stages of development of human long bones. The character of this tissue has been described in a previous paper (Smith 1960), and it is illustrated in the transverse section of decalcified bone in Figure 8. The primary osteones (E) are longitudinal tracts of parallel-fibred bone, oval or sausage-shaped in cross-section, which surround a variable number of longitudinal vascular canals. Adjacent primary osteones are separated by plaques of woven fibred bone $(G)$, most of which extend along longitudinal planes which are concentric with the surfaces of the whole bone. These main plaques are united here and there by shorter radial plaques of the same tissue $(\mathrm{H})$.

On microradiography (Fig. 9) young primary osteone bone exhibits two grades of mineralisation. As has been noted by Ponlot (1960) the woven-fibred plaques are highly mineralised soon after their formation $(\mathrm{G}$ and $\mathrm{H})$. On the other hand the primary osteones $(\mathrm{E})$ 
have a comparatively low inorganic content, and it is only as the tissue ages that it increases towards parity with the adjacent plaques.

Carbonisation of similar tissue (Fig. 10) shows that by and large the organic content is inversely proportional to the degree of mineralisation (Smith 1963). The highly calcified woven-fibred plaques ( $G$ and $H)$ contain little organic material, whereas the organic concentration in the primary osteones (E) is relatively high. Again it is only as the tissue ages

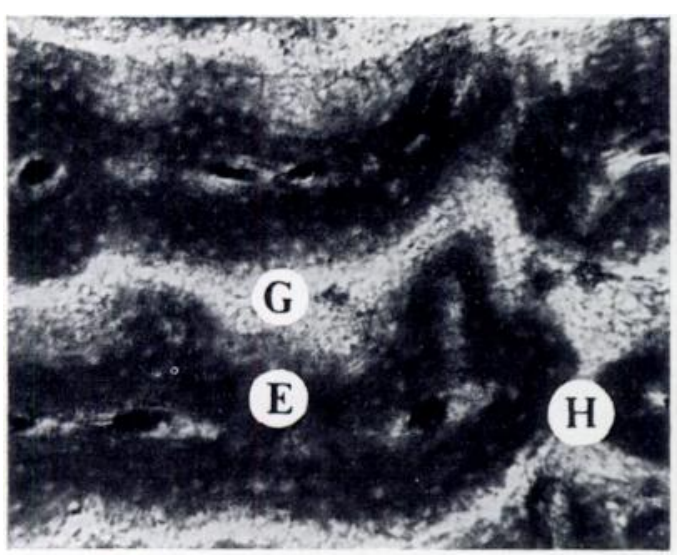

Fig. 10

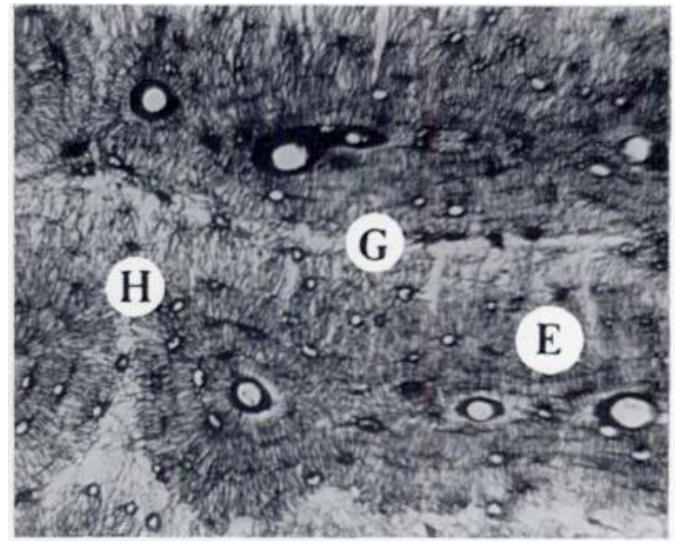

Fig. 11

Figure 10-Carbonised preparation of a ground section of the femur of a pig, labelled as in Figure 8. ( . 110.) Figure 11-Transverse section of a decalcified ox metatarsus, labelled as in Figure 8. (Mulligan's silver method, $\times 125$. .)

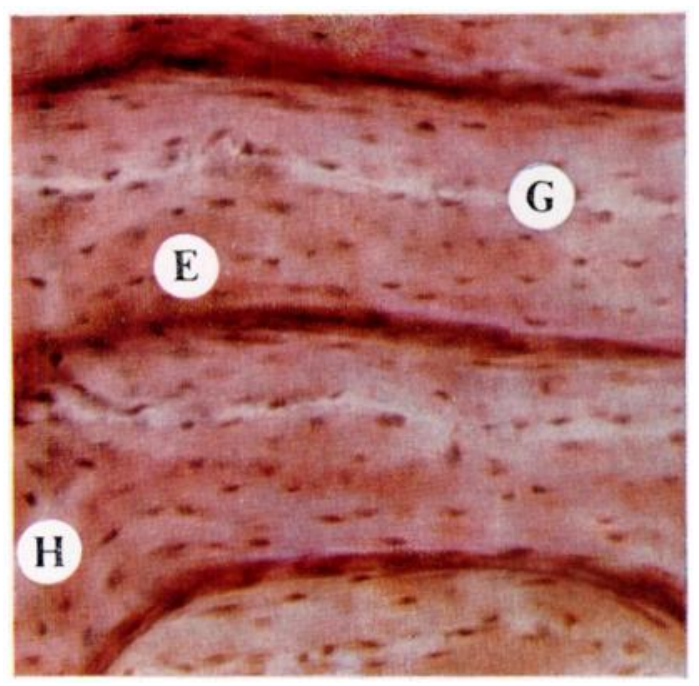

FIG. 12

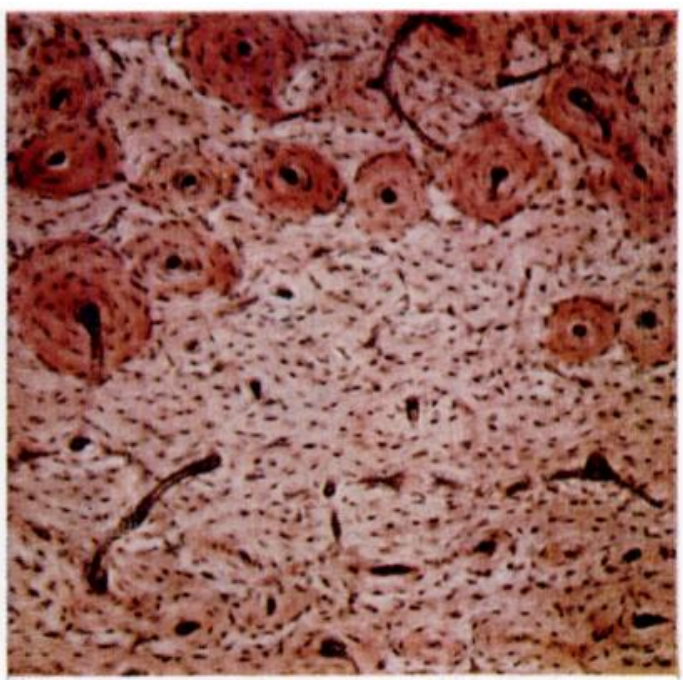

FIG. 13

Figure 12-Sudan 4 impregnation of a ground section of an ox metatarsus. E primary osteone. G wovenfibred plaque. $\mathrm{H}=$ radial plaque. ( . 120.) Figure 13-Sudan 4 impregnation of a ground section of the femur of a dog. ( 75.)

that the organic content of the primary osteones decreases towards parity with the adjacent plaques.

Figure 11 shows a decalcified section of similar bone stained by Mulligan's method. As Lacroix (1960) has noted previously, the lacunae and canaliculi pervade the primary osteones $(E)$ as a rich plexus, whereas the woven-fibred plaques $(G$ and $H)$ are practically devoid of both elements. 
The water content of a similar region of young primary osteone bone is shown in Figure 12 in a ground section impregnated with Sudan 4 . The woven-fibred plaques ( $G$ and $H$ ) contain little if any pigment, and, although this is due in part to the virtual absence of canaliculi, it also indicates a very low water content in the bone tissue proper in these regions. In the primary osteone (E) the canaliculi can just be discerned at this magnification and they with the lacunae and the vascular spaces are heavily impregnated. The bone tissue proper is also brightly tinted and therefore contains a considerable quantity of water.

Thus in primary bone, as in secondary bone, highly mineralised regions have low contents of both organic material and water, whereas poorly mineralised regions are relatively rich in both of these fractions.

Mixed primary and secondary bone-As primary bone ages, changes occur in the proportions of its inorganic, organic and water fractions. Eventually it undergoes focal resorption and, in the resorption cavities so formed, secondary osteones are formed. Such secondary osteones are always younger than the primary bone surrounding them, and as a result they exhibit different proportions of their three constituent fractions.

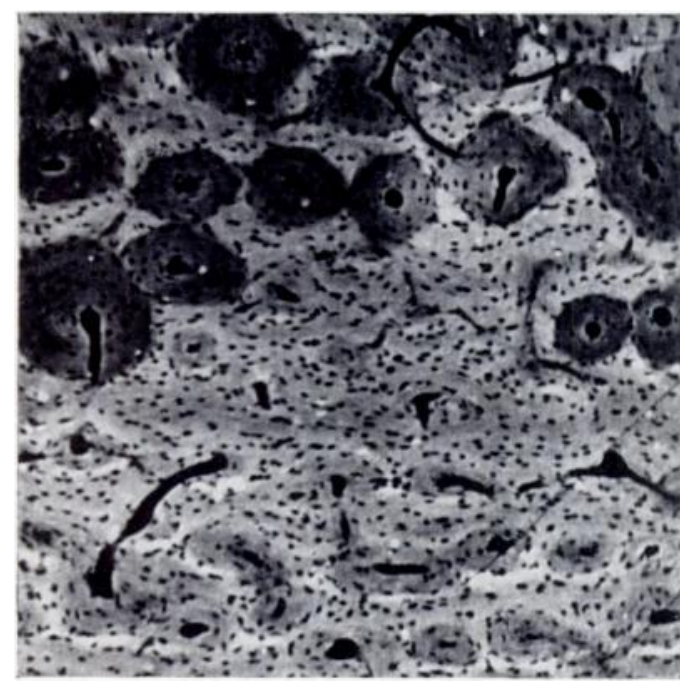

FiG. 14

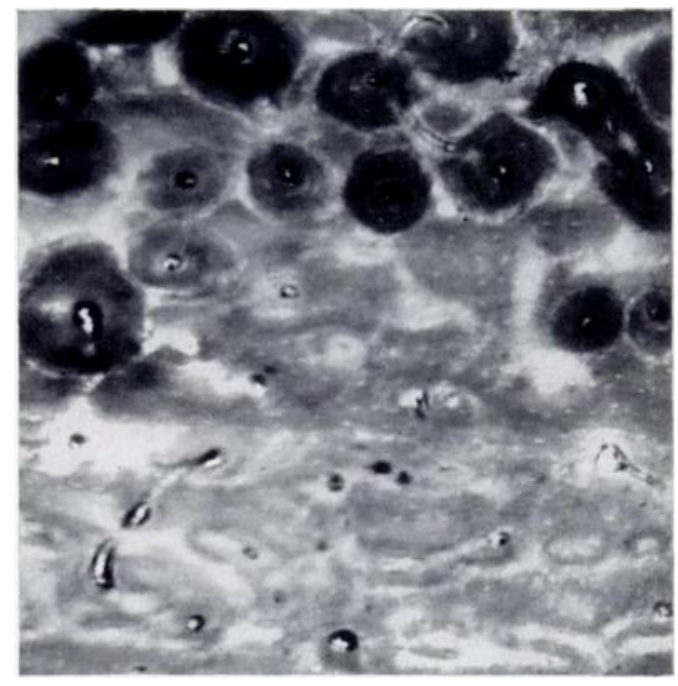

Fig. 15

Figure 14-Microradiograph of the field shown in Figure 13. (.75.) Figure 15-Carbonised preparation of the field shown in Figures 13 and 14. (75.)

Figure 14 is a microradiograph of an area in which the processes noted above are occurring. The lower half of the field consists of old primary osteone bone. The primary osteones, being older than those illustrated in Figure 9, have a higher mineral content, and consequently the contrast between these osteones and the adjacent woven-fibred plaques, though still evident, is less distinct. In the upper half of the field a large number of secondary osteones have formed within resorption cavities, and it is evident that this comparatively young secondary tissue is poorly mineralised in comparison with the older primary osteone bone which surrounds it.

The organic content of the same region is shown as a carbonised preparation in Figure 15. In the old primary osteones in the lower half of the field the high organic content noted in young primary osteones (Fig. 10) is no longer evident, because the organic content has decreased with age until it approaches that of the adjacent woven-fibred plaques. On the other hand the majority of the young secondary osteones in the upper half of the field have a high organic content, which is comparable to that of the young secondary osteone in Figure 2 and the young primary osteones in Figure 10. 
The water content of the same region is shown by Sudan 4 impregnation in Figure 13 . Contrasting the old primary osteones in the lower part of this field with the young primary osteones in Figure 12, it is evident that their water content is distinctly lower. On the other hand the water content of the young secondary osteones in the upper part of the field is much higher than that of the surrounding primary tissue, and is of the same order as that in the young secondary osteones in Figures 5 to 7, and in the young primary osteones in Figure 12.

\section{DISCUSSION}

It seems evident from these observations that, in most regions of bone tissue proper, both the water content and the organic content are inversely proportional to the mineral concentration. In some special regions, such as the woven-fibred plaques of primary bone and the reversal lines of secondary bone, the association of a high mineral content with very low organic and water contents is achieved while the tissue is comparatively young. But in most regions, including primary and secondary osteones, the mineral content is low in young bone and rises at a progressively diminishing rate over a period of years (Jowsey 1958) to the high mineral concentration characteristic of old tissue. This progressive mineralisation occurs at the expense of both the organic and water fractions so that young bone is characterised by a high content of both water and organic material, whereas old tissue exhibits low concentrations of both these fractions. Moreover, as might be expected, low water and organic contents tend to be associated with lower rates of ionic exchange in the tissue and with a paucity of canaliculi.

The lack of an exact relationship between the total water content of the tissue and the water content demonstrated by the present method has already been noted. The method is to be regarded as giving an assessment of the relative amounts of free water and collagenbound water in adjacent areas of bone. At the lowest levels of water content another difficulty arises. As the water content of bone is reduced, a stage is eventually reached when some or all of the spaces occupied by water have dimensions of a molecular order. In these circumstances the ability of the pigment solution to enter, and thus demonstrate, the water space becomes a question of the size of the pigment molecule. The molecular weight of Sudan 4 is 380 (Gurr 1960), and it appears probable, therefore, that impregnation of bone with this pigment will fail to demonstrate water contents below a certain level: in other words all areas with water contents below this critical level will appear colourless. Although this critical level is not known it is probable that it is very low.

The impregnation of bone by pigments, as described in this paper, is somewhat similar to the staining of fresh ground section with basic fuchsin described by Frost (1960b), but the interpretation of the results is very different. Frost argued that the regions which were stained by his method were abnormal and represented a peculiarity of skeletal mineralisation which he termed "feathering." In the present investigation pigmentation of areas of bone has been observed in every section examined, and indeed in Frost's own series ninety-one of his ninetyfive specimens exhibited stained or feathered areas. It is difficult to correlate such an incidence with the concept of abnormality. According to Frost's description, fuchsin staining, or feathering, occurs more frequently in complete osteones than in interstitial areas or cement lines, and more intensely in regions which are poorly mineralised than in those which are highly mineralised. Nevertheless, he holds that the stained areas do not represent areas of young bone but of old bone which has failed to become mineralised. The great bulk of autoradiographic and microradiographic studies in recent years and in particular the work of Lacroix (1960) runs contrary to such a concept: indeed it is considered that all the feathered areas illustrated in Frost's paper are recognisable as young osteones, either from the size of their vascular canals or from their spacial relationship to the surrounding units of bone.

VOL. 46 B, NO. 3, AUGUST 1964 
It is considered therefore that the pattern of staining or impregnation observed in ground sections of bone cannot be satisfactorily explained by the concept of an abnormal process of mineralisation. On the contrary it is submitted that the correlation of pigmentation with water content, which has been suggested in this paper, is a more reasonable explanation.

\section{SUMMARY}

1. A method is described by which the relative water contents of adjacent microscopic regions of bone can be assessed.

2. The water content is correlated with the inorganic and organic contents in regions of different age.

3. The results suggest that the age increase in the mineralisation of bone occurs at the expense of both the organic and water fractions.

I am grateful to Mr J. Brown and Mr R. Stuart of this Department for the preparation of the illustrations. The film used in microradiographic examinations was kindly provided by Messrs Kodak Ltd.

\section{REFERENCES}

EAStOE, J. E. (1955): The Amino Acid Composition of Mammalian Collagen and Gelatin. Biochemical Journal, 61, 589.

Edsall, J. T. (1953): The Size, Shape and Hydration of Protein Molecules. In the Proteins. Edited by H. Neurath and K. Bailey. New York: Academic Press Inc.

Frost, H. M. (1960a): Micropetrosis. Journal of Bone and Joint Surgery, 42-A, 144.

Frost, H. M. (1960b): A New Bone Affection: Feathering. Journal of Bone and Joint Surgery, 42-A, 447.

GURR, E. (1960): Encyclopaedia of Microscopic Stains. London: Leonard Hill Books Limited.

Jowsey, J. (1958): Quoted by R. A. Robinson in Bone as a Tissue. Edited by K. Rodahl, J. T. Nicholson and E. M. Brown. London: McGraw-Hill.

LACrolx, P. (1960): $\mathrm{Ca}^{45}$ Autoradiography in the Study of Bone Tissue. In Bone as a Tissue. Edited by K. Rodahl, J. T. Nicholson and E. M. Brown. London: McGraw-Hill.

Mulligan, J. H. (1958): The Canalicular System of Bone. Journal of Anatomy, London, 92, 672.

Mulligan, J. H. (1964): Personal Communication.

Neuman, W. F., Toribara, T. Y., and Mulryan, B. J. (1953): The Surface Chemistry of Bone. ViI. The Hydration Shell. Journal of the American Chemical Society, 75, 4,239.

Neuman, W. F., and Neuman, M. W. (1958): The Chemical Dynamics of Bone Mineral. Chicago: University of Chicago Press.

Ponlot, R. (1960): Le radiocalcium dans l'étude des os. Thesis, University of Louvain. Brussels: Arscia.

Robinson, R. A. (1960): Chemical Analysis and Electron Microscopy of Bone. In Bone as a Tissue. Edited by K. Rodahl, J. T. Nicholson and E. M. Brown. London: McGraw-Hill.

Sissons, H. A. (1962): Age Changes in the Structure and Mineralization of Bone Tissue in Man. In Radioisotopes and Bone. Edited by F. C. McLean, P. Lacroix and A. M. Budy. Oxford: Blackwell Scientific Publications.

Sмiтн, J. W. (1960): Collagen Fibre Patterns in Mammalian Bone. Journal of Anatomy, London, 94, 329.

Smith, J. W. (1963): Age Changes in the Organic Fraction of Bone. Journal of Bone and Joint Surgery, 45-B, 761.

Smith, J. W., and Walmsley, R. (1959): Factors Affecting the Elasticity of Bone. Journal of Anatomy, London, 93, 503.

Tristram, G. R. (1953): In The Proteins. Edited by H. Neurath and K. Bailey. New York: Academic Press Inc. 